\title{
Strukturen ungleichen Erfolgs. Winner- take-all-Konzentrationen und ihre sozialen Entstehungskontexte auf flexiblen Arbeitsmärkten
}

\author{
MPIfG Journal Article \\ Mark Lutter: Strukturen ungleichen Erfolgs: Winner-take-all-Konzentrationen und ihre sozialen Entstehungskontexte auf flexiblen \\ Arbeitsmärkten. In: Kölner Zeitschrift für Soziologie und Sozialpsychologie 65(4), 597-622 (2013). \\ VS Verlag für Sozialwissenschaften \\ The original publication is available at the publisher's web site: http://dx.doi.org/10.1007/s11577-013-0238-1 \\ The MPIfG Journal Articles series features articles by MPIfG researchers and visiting scholars published in peer-reviewed journals. \\ Max Planck Institute for the Study of Societies (MPIfG) Cologne | www.mpifg.de
}

Zusammenfassung: Wie entstehen Erfolgskonzentrationen? Während das „Winner-take-all“Phänomen bisher als Konzentrationsprozess auf der Nachfrageseite durch massenhaft gleichförmige Kaufentscheidungen der Konsumenten begriffen wurde, sind Bedingungen und Konstellationen auf der Anbieterseite wenig berücksichtigt worden. In diesem Beitrag werden sechs Ansätze diskutiert, die das Potenzial einer soziologischen Erklärung des Winner-take-all-Phänomens ausloten. Jeder der Ansätze versucht, Erfolgsungleichheiten aus den sozialen Strukturen heraus zu erklären, in die die Akteure auf dem Arbeitsmarkt eingebettet sind. Der Beitrag versteht sich als erster Zugang zu einem in der Soziologie zwar noch wenig erforschten, doch wichtigen Phänomen sozialer Ungleichheit und soll den Raum für zukünftige empirische Studien öffnen.

Schlüsselwörter: Erfolgskonzentrationen · Winner-take-all · Projektarbeitsmärkte ·

Matthäus-Effekte $\cdot$ Heterachie $\cdot$ Netzwerke $\cdot$ Nischentheorie $\cdot$ Statushomophilie

\section{Structures of unequal success. The social contexts of emerging winner-take-all-concentrations on flexible labor markets}

\begin{abstract}
How does success accumulate? While the winner-take-all phenomenon has been viewed as a process of accumulating demand that results from a huge number of consumers making the same purchase decision, the conditions and interactions on the supply side have received scant attention in the literature. This paper investigates six ways sociology could contribute toward shedding light on the winner-take-all phenomenon, all of which seek to explain unequal success by examining the social structures of the labor market in which the actors are embedded. The author takes a preliminary, exploratory look from a sociological perspective at an aspect of inequality that is socially significant, yet poorly understood. The approaches presented open the way for future empirical study.
\end{abstract}

Keywords: Concentration of success - Winner-take-all - Project-based labor markets · Matthew-effects · Heterachy $\cdot$ Networks $\cdot$ Niche theory $\cdot$ Status homophily

(C) Springer Fachmedien Wiesbaden 2013

\section{Lutter $(\bowtie)$}

Max-Planck-Institut für Gesellschaftsforschung,

Paulstr. 3, 50676 Köln, Deutschland

E-Mail: lutter@mpifg.de 


\section{Einleitung}

In den letzten Jahrzehnten haben soziale Ungleichheiten in den westlichen Industrienationen wieder zugenommen. Die Zunahme ist allerdings weniger auf eine steigende allgemeine Einkommensungleichheit breiter Schichten zurückzuführen, sondern auf Konzentrationsprozesse im oberen 1\%-Perzentil der Einkommen (McCall und Percheski 2010, S. 333; Western et al. 2008, S. 905). Es handelt sich also um Zuwächse nach dem „Winner-take-all“-Prinzip: Heute befindet sich erheblich mehr Vermögen in den Händen einer kleineren Anzahl Privilegierter. Neben der Vererbung von Vermögen (Beckert 2004; Szydlik 1999, 2004; Szydlik und Schupp 2004), bildungs- und herkunftshomogamen Familienstrukturen (Blossfeld 2009; Western et al. 2008), starken Einkommenszuwächsen in der Finanzwelt und den Unternehmensvorständen (DiPrete et al. 2010; Neckel 2010) sowie einschlägiger politischer Reformprozesse (Hacker und Pierson 2010, S. 168) gründet sich ein Teil der Zunahme auf Erfolgsungleichheiten in jenen flexiblen Arbeitsmärkten, die durch „Superstars“ dominiert sind. Superstarphänomene lassen sich in unterschiedlicher Ausprägung in verschiedenen Branchen beobachten: im Management, in der Wissenschaft, auf Arbeitsmärkten für Rechtsanwälte, Architekten, Journalisten, Politiker, Psychologen oder Medizinern (Frank und Cook 1995). Prävalent sind erfolgskonzentrierte Arbeitsmärkte aber vor allem in den Kultur-, Medien- und Kreativindustrien (Cowen 2000; Frank und Cook 1995; Menger 1999; Rosen 1981).

Wie erklärt sich das Zustandekommen von Winner-take-all-Konzentrationen auf diesen Arbeitsmärkten? Verdeutlicht werden soll das Phänomen zunächst anhand eines Beispiels aus der Filmbranche. Die Gagen der erfolgreichsten Filmschauspieler betragen ein Vielfaches des durchschnittlichen Einkommens; zugleich hat die Mehrheit der Filmschaffenden mit sehr prekären Karrierebedingungen zu kämpfen (Bührmann und Dierschke 2012). Eine Umfrage unter deutschen Schauspielern konkretisiert dies mit Zahlen: Knapp über $60 \%$ waren innerhalb eines Zeitraums von zwei Jahren weniger als sechs Wochen beschäftigt; der jährliche Bruttoverdienst lag für die Hälfte aller befragten Schauspieler bei unter $20000 €$ (Bührmann et al. 2010, S. 6-10). Diese Summe entspricht in etwa der Gage, die die Spitzenverdiener unter den deutschen Schauspielern für zwei Drehtage erhalten. ${ }^{1}$

Nicht nur die Einkommen sind ungleich verteilt: Das Gros der Produktionen wird von einer erfolgreichen Minderheit dominiert. Faulkner und Anderson (1987, S. 894) zeigen, dass 7\% der Regisseure in Hollywood $40 \%$ aller Spielfilme produzieren; einen einzigen Film produzieren dagegen fast zwei Drittel. Über $75 \%$ der Regisseure sind im Verlauf ihrer Karriere an nicht mehr als zwei Hollywoodfilmen beteiligt.

$\mathrm{Zu}$ kennzeichnen sind Winner-take-all-Märkte durch zwei Grundelemente. Erstens vereinen relativ wenige Marktakteure die meisten Erfolgsanteile auf sich. Die Gewinne können derart konzentriert sein, dass die Summen einiger weniger praktisch dem gesamten Volumen des Marktes entsprechen. Zweitens herrscht ein permanentes Überangebot an Akteuren und Talenten, die in diese Märkte hineindrängen und zur Übersättigung

1 So verlangt der deutsche Schauspieler Götz George 9000 Euro pro Drehtag (Süddeutsche Zeitung vom 2. Juli 2009, www.sueddeutsche.de/panorama/top-verdiener-in-der-deutschen-filmbranche-1.82643-8). 
der Nachfrage beitragen. Die Überlebenschancen sind damit gering. Die Mehrheit der Akteure ist mit prekärer Beschäftigung, Unterbeschäftigung, branchenferner Arbeit und Arbeitslosigkeit konfrontiert (Menger 1999, S. 545). Oft bleibt nach einiger Zeit der Erfolglosigkeit und dem Eingestehen derselben nur der Weg, auf alternative Berufswege auszuweichen.

Das Zustandekommen solcher Verteilungen zu erklären, stellt insbesondere für die ökonomische Standardtheorie eine Herausforderung dar, weil unter perfekten Marktbedingungen keine konzentrierten Erfolge zu erwarten wären (Borghans und Groot 1998, S. 569). Ganz im Gegenteil resultiert für Frank und Cook (1995, S. 125 ff.) das Starsystem in der sozial ineffizienten Allokation von Talent. Die exorbitanten Gewinne der wenigen Topperformer locken ständig mehr junge Talente an, als die Nachfrage danach bedienen kann. Somit müssen viele nach Alternativen suchen, weil die lukrativen Positionen besetzt und unerreichbar sind. Da die meisten Akteure allerdings nach Jahren der Fokussierung für Berufswege zweiter Wahl kein marktrelevant zugeschnittenes Humankapital besitzen und zudem denen unterlegen sind, die das haben, stellt das Alles-odernichts-Prinzip dieser Arbeitsmärkte ein gesellschaftlich nichteffizientes Marktergebnis her: Es verschwendet Talent. Beispielsweise konkurrieren jedes Jahr Hunderte Absolventen der Musikhochschulen um die wenigen Positionen in den Ensembles der Orchester. Die intensive musikalische Ausbildung erfordert ein hohes Maß der Spezialisierung an einem Instrument. Die meisten Musikschüler müssen ihr Instrument von der Kindheit an mehrere Stunden täglich spielen, um das für Hochschulen nötige Aufnahmeniveau zu erreichen. Gelingt eine Festanstellung nach dem Studium nicht, sind viele aufgrund mangelnder äquivalenter Alternativen gezwungen, ihre professionellen Musikerkarrieren zu beenden, noch bevor sie überhaupt begonnen haben. ${ }^{2}$ Hätten sie ihr Talent dagegen von vornherein auf gesellschaftlich besser nachgefragte Arbeitsmärkte verlegt, bliebe ihr Humankapital nicht fehlgeleitet, sondern dem Bedarf optimal angepasst.

Das Starsystem bewirkt nicht nur die Fehlleitung von Talent, sondern verursacht darüber hinaus Kostenineffizienzen auf der Produktionsseite. Denn die exorbitanten Gehälter der Stars können zur Überschuldung oder Verlusten führen. Vereine wie FC Chelsea London können ihre hohen Spielergehälter zahlen, weil ein Ölmilliardär den Verein ohne ökonomische Gewinnabsichten führt und die verlustreichen Investitionen privat tätigt. In Hollywood zählen die Gagen der Stars mittlerweile zu den größten Kostenfaktoren einer Produktion und können häufig nicht mehr über Einnahmen an den Kinokassen amortisiert werden. Daher stellen die meisten Filme Verlustgeschäfte dar, die über Sekundärmärkte (zum Beispiel Merchandising, Lizenzierungen, Unterhaltungselektronik) ausgeglichen werden müssen. Der Film ist lediglich ein riesiger, kostenintensiver Werbeträger (siehe ausführlich: Leaver 2010; Meier 2008).

Wie lassen sich die Bedingungen des Erfolgs auf diesen Arbeitsmärkten erklären? Wie kommt es zu den extremen Unterschieden? Bisherige Ansätze haben das Winner-take-

2 Vgl. kürzlich in der F.A.Z. unter der Überschrift „Mit dem Instrument in die Sackgasse“ (F.A.Z. vom 7. April 2012). Ein ähnliches Dossier veröffentlichte Die Zeit unter dem Titel: „Das Vorspiel. In Berlin treten junge Kontrabassisten aus der ganzen Welt gegeneinander an: Ein Wochenende lang spielen sie um die einzige freie Stelle des Konzerthausorchesters - und um ihre Zukunft" (Die Zeit vom 17. Februar 2011). 
all-Phänomen als Nachfragekonzentration verstanden (Adler 1985; Rosen 1981) und im Wesentlichen auf die Kaufpräferenzen rationaler Konsumenten oder Nachfragern nach Talenten zurückgeführt, die ihre Entscheidungen so stark auf einige wenige „Bestseller“ richten, dass extreme Erfolgskonzentrationen möglich werden (vgl. kürzlich dazu umfassend: Keuschnigg 2012a, b). Die Anbieterseite, und damit die Frage, welche Konstellationen Erfolg auf der Seite der Akteure des Arbeitsmarktes bedingen, ist bislang kaum Gegenstand dieser Forschung gewesen.

Darüber hinaus sind die bisherigen Ansätze disziplinär nahezu ausschließlich im individualistischen Paradigma der ökonomischen Theorie angesiedelt. Obwohl einige der Ansätze durch Modellierung und Hinzunahme sozialer Ansteckungsprozesse gewissermaßen „soziologisiert“ worden sind (Keuschnigg 2012a), so entstehen Winner-take-all-Strukturen aus dieser Perspektive aus der Vielzahl individueller Entscheidungen rationaler Akteure. Die hierarchische Struktur des extrem konzentrierten Erfolgs auf diesen Arbeitsmärkten ist somit als „emergentes“ Resultat der individuellen Präferenzen der Nachfrager sowie aus den Talenten und Qualitäten der angebotenen Produkte zu verstehen. Soziale Ungleichheit ist aus dieser Sicht das quasinatürliche Ergebnis mikro-motivationaler Prozesse.

Für strukturelle soziologische Ansätze dagegen bestehen die ungleichen Verteilungen nicht als emergentes, aufgekommenes Phänomen, sondern erklären sich aus den unterschiedlichen sozialen Positionen, die Akteure bekleiden, und den sozialen Strukturen, die sie umgeben (vgl. Gould 2002, S. 1144). Derartige Erklärungsansätze sind, auf das Winner-take-all-Phänomen bezogen, allerdings bislang rar. ${ }^{3}$ Beispielsweise sind soziale Netzwerkstrukturen noch nicht systematisch in die Erklärungsversuche eingeschlossen worden. Somit bleibt unklar, inwiefern soziale Strukturen, in die die individuellen Entscheidungen eingebettet sind, Unterschiede im Erfolg erklären können.

Im vorliegenden Beitrag soll das Augenmerk daher primär auf diese soziologischstrukturellen Erklärungsansätze gelegt und der Frage nachgegangen werden, welche geeignet sein könnten, Erfolgskonzentrationen auf der Seite der Anbieter zu erklären. Der Fokus soll auf der Kreativbranche und dem Arbeitsmarkt für Künstler liegen (Becker 1982; Caves 2000; Florida 2002; Koppetsch 2006, 2008; Menger 1999, 2009). Diese Ausrichtung geschieht aus mehreren Gründen. Zum einen bietet sich die Kreativbranche als Musterbeispiel an, da sich das Phänomen dort häufig und in besonderer Schärfe beobachten lässt. Darüber hinaus knüpft der Beitrag an bestehende ökonomische Erklärungsansätze an, die primär anhand dieser Branche entwickelt wurden. Drittens erzeugt es analytische Kohärenz, wenn die hier diskutierten Ansätze an einem konkreten empirischen Fall orientiert bleiben. Es steht weiterer Forschung offen, diese auf weitere Felder zu übertragen. ${ }^{4}$

3 Es gibt zwar in der Managementwissenschaft die sogenannte Erfolgsfaktorenforschung, die aber unter anderem wegen ihrem Reduktionismus umstritten ist, der soziale Strukturen, in die Akteure eines Unternehmens oder einer Branche eingebettet sind, systematisch unbeachtet lässt (March und Sutton 1997; Nicolai und Kieser 2002).

4 Für soziologische Ansätze, die sich auf Erklärungen des Winner-take-all-Phänomens unter Managern beziehen, sei auf weitere interessante Forschung verwiesen, wie DiPrete et al. (2010), Godechot (2008, 2012) und Rost (2010). 
Ziel dieses Artikels ist es, soziologische Ansätze auszuleuchten, mit denen Erfolgsbedingungen und damit mögliche Konzentrationsprozesse aus den sozialen Strukturen heraus erklärt werden können, in die die Akteure des Arbeitsmarktes auf der Anbieterseite eingebettet sind. Ausgehend von einer Diskussion der wichtigsten wirtschaftswissenschaftlichen Erklärungsansätze schlussfolgert die Studie zunächst, dass sowohl Rosen (1981) als auch Adler (1985) sowie diffusionstheoretische Erweiterungen (unter anderen Banerjee 1992) keine ausreichende Erklärung dafür anbieten, welche anfänglichen Mechanismen die Entstehung von Erfolg bedingen (siehe auch Keuschnigg 2012b, S. 145 ff.). Die Erklärungen setzen erst dann ein, wenn bereits eine hinreichende Masse an Nachfragern ihre Konsumentscheidung angeglichen hat. Diffusionstheoretische Ansätze modellieren die weitere Zunahme von Nachfragekonzentration. Welche ursächlichen Primärmechanismen jedoch den Erfolg bedingen, ist darin nicht zu erklären. Genau hier setzt der vorliegende Artikel an und versucht, soziologische Konstellationen und Bedingungen auf der Seite der Anbieter heranzuziehen, mit denen die Entstehungsbedingungen ungleichen Erfolgs erklärt werden können.

Insgesamt werden sechs Ansätze aus der neueren Wirtschafts- und Organisationssoziologie und der soziologischen Ungleichheitsforschung herangezogen. Die Ansätze umfassen 1) Erfolgsbenachteiligungen aufgrund von askriptiven Ungleichheitsprozessen und allokativen Opportunitätsstrukturen für Diskriminierungen, 2) kumulierte Vorteile oder „Matthäus-Effekte“, 3) Netzwerkstrukturen und die „Stärke schwacher Beziehungen“, 4) Nischenabdeckung und Rollenausdehnung als Erfolgsfaktoren, 5) organisationale Heterarchie in kreativen Gruppenprozessen und schließlich 6) Statushomogamien bei der Zusammenstellung kreativer Teams.

Der Aufsatz versteht sich als ein wirtschaftssoziologischer Beitrag zur sozialen Ungleichheitsforschung auf flexiblen Arbeitsmärkten, in denen hochgradig konzentrierte Ungleichverteilungen von Erfolg bestehen. Die Arbeit liefert eine erste Bestandsaufnahme, wie soziologische Ansätze zur Erklärung von Erfolg genutzt werden können. Sie bildet damit einen Ausgangspunkt für weitere empirische wie theoretische Analysen.

Aufgebaut ist der Aufsatz wie folgt: Zunächst wird kurz der bisherige Forschungsstand zur Erklärung extremer Erfolgskonzentrationen referiert (Abschn. 2). Im Anschluss daran wird jeder der sechs soziologischen Ansätze vorgestellt (Abschn. 3). Es wird zuerst die allgemeine theoretische Logik des Ansatzes betrachtet, anschließend die bisherige relevante empirische Forschung diskutiert. Jeder Ansatz schließt mit einer Hypothese, die die Erklärungen jeweils zusammenfasst. Im Schlussteil (Abschn. 4) werden die wichtigsten Ergebnisse zusammengetragen und ihre Implikationen für zukünftige (empirische) Forschung ausgelotet. Der Artikel endet mit dem kurzen Anriss einer Reihe weiterer Forschungsfragen, die dafür plädieren, für eine mikrofundierte Erklärung des Winner-take-all-Phänomens die Untersuchung der Karrieremotive der Akteure in den Vordergrund zu rücken.

\section{Forschungsstand: Winner-take-all-Märkte als Nachfragekonzentration}

Das Winner-take-all-Phänomen wurde erstmalig durch Sherwin Rosen (1981) einer systematischen Erklärung unterzogen und später wesentlich durch Adler (1985) erweitert. 
Beide Ansätze konzentrieren ihre Erklärungen des Phänomens auf die Nachfrageseite der Kultur- und Medienwirtschaft. Weitere Autoren haben diese Modelle modifiziert, aber in ihren Grundlagen nicht wesentlich verändert (Franck und Nüesch 2012; Lazear und Rosen 1981; MacDonald 1988; Rosen 1986). Es ist daher sinnvoll, die zwei grundlegenden Ansätze von Rosen und Adler näher zu betrachten.

Unter der Annahme rationaler Konsumenten und vollständiger Informationen identifiziert Rosen zwei Ursachen für die Entstehung des Superstarphänomens auf Kulturgütermärkten (Rosen 1981, S. 846 f.). Zum einen wird die Nachfragekonzentration auf Effekte unvollständiger Substitutionen der auf dem Markt angebotenen Güter zurückgeführt, zum anderen auf die kostengünstige massenhafte Vervielfältigung der kulturellen Güter, die im Zuge technischer Entwicklungen zunehmend möglich wird.

Beide Sachverhalte wollen kurz erläutert werden. Die Grundannahme des Ansatzes ist, dass sich rationale Konsumenten bei ihrer Wahl zwischen Gütern der gleichen Kategorie für die Produkte oder Leistungen entscheiden, die mit der besten Qualität versehen sind. Ausschließlich diese wollen sie nachfragen und konsumieren. Die typischerweise in den Kultur- und Kreativindustrien hergestellten Güter besitzen die Eigenschaft, keine oder unvollständige Substitutionsmöglichkeiten im Hinblick auf ihre Qualität zu bieten. Mit anderen Worten: Produkte von minderer Qualität bilden nur sehr mäßigen Ersatz für Güter mit Spitzenqualität. Beispielsweise addiert sich das Lesen mehrerer schlechter Romane nicht auf ein hochwertiges Buch; die musikalische Darbietung vieler mittelmäBiger Orchester ersetzt nicht die Qualität des Spitzenensembles.

Die unvollständige Substituierbarkeit allein reicht allerdings noch nicht aus, um die extremen Konzentrationen auf diesen Märkten hinreichend zu erklären. Es muss ein zweiter Aspekt hinzukommen: die technische Reproduzierbarkeit künstlerischer Werke. Diese sorgt für eine weltweite Verbreitung bei gleichbleibenden Kosten. Erst darüber wird der Zugang zur bestmöglichen Qualität über lokale Grenzen hinweg fast unbegrenzt ermöglicht. So sind Musikdarbietungen heutzutage nicht mehr an einen Konzertort gebunden. Auf Ton- und Bildträger gepresst, digital verarbeitet und angeboten, können sie weltweit ohne wesentliche Mehrkosten verbreitet und nachgefragt werden. Die Nachfrage konzentriert sich unter diesen Bedingungen vollständig auf die besten der am Markt verfügbaren Produkte, Talente und Darbietungen. Die Anbieter an der Spitze vereinen die gesamte Nachfrage auf sich und beginnen, den Marktabsatz zu dominieren. Sie können Premiumbeträge für ihre Güter und Dienstleistungen verlangen, für die die Nachfrager zu zahlen bereit sind.

Eine wesentliche Voraussetzung dieses Erklärungsmodells ist neben der Präferenz der Nachfrager für die beste Qualität ihre vollständige Information darüber, welche Angebote tatsächlich Spitzenqualität besitzen. Während dies im Sport noch objektiv zu ermitteln ist, ${ }^{5}$ besitzen Güter aus künstlerischer Arbeit grundsätzlich streitbare Qualitäten. So erschließt sich die Qualität eines Kunstwerks nicht mehr, wie noch beim HandwerkerKünstler im Altertum und Mittelalter (Müller-Jentsch 2005, S. 162), anhand der handwerklichen Arbeitszeit oder den Kosten des Verwendung findenden Materials. Auch nicht

5 So findet nach Keuschnigg (2012b, S. 88) das Modell von Rosen für Superstarphänomene im Sport die beste Anwendung. 
die reine Seitenzahl oder die Feinstruktur des Papiers sind ausschlaggebend für die Qualität eines Romans, ebenso wenig Inhalt und Form der erzählten Geschichte.

Anhand objektiver oder objektivierbarer Kriterien lässt sich ihre Qualität nur grob ermitteln. Aufgrund der Singularität der angebotenen Produkte entzieht sich Qualität vielmehr der Objektivierbarkeit (Karpik 2010). Stattdessen ist sie zuhöchst variabel, weil sie erst durch soziale Akteure im Feld der Kunst oder der Literatur auszuhandeln ist und erstritten, definiert und hergestellt werden muss (Beckert und Aspers 2011; Beckert und Musselin 2013). Damit können Qualitätsurteile Moden, Meinungen und Marktmächten unterliegen, sind nie universell gültig, sondern abhängig von Kontext und Zeit. Entsprechend zeigt eine Studie über den Kunstmarkt, wie das, was als wertvoll gilt, durch Kritikerurteile im Feld der Kunst sozial konstruiert wird und als Qualitätsmarker die subjektiven Urteile der Nachfrager leitet (Beckert und Rössel 2004, S. 34).

Künstlerische Güter sind zudem mit Lernkosten verbunden und offenbaren ihre Qualität erst nach einiger Zeit der Beschäftigung mit ihnen oder dem Genre, dem sie entstammen. Ihre Qualität muss erst zu schätzen gelernt werden. Bücher sind beispielsweise Erfahrungsgüter, deren Qualität sich für die Konsumenten nicht beim Kauf, sondern erst nach ihrem Konsum zeigt (Keuschnigg 2012b, S. 16). Die Qualität einer komplexen, frei atonalen Aufführung eines Ensembles im Bereich Neuer Musik erschließt sich dem Konsumenten nur durch ein entsprechend geschultes Ohr und das angelernte Wissen über die historische und musikalische Bedeutung der Zwölftonmusik. Kurz, es bedarf eines notwendigen Maßes an kulturellem Kapital, das die Qualitätsbeurteilung erst ermöglicht. Die individuelle Konsumentscheidung ist damit in hohem Maße sozial vorstrukturiert (Bourdieu 1985; Rössel 2009; Rössel und Bromberger 2009).

Der Ansatz von Rosen ist also nur im theoretischen Modell rationaler und vollständig über Qualitätsmaßstäbe informierter Akteure zu verstehen. Empirisch ist dies eine sehr strikte Annahme. Tatsächlich zeigt sich sogar, dass oft jene Angebote Erfolg haben, die von anerkannten Experten nicht zur besten Qualität gezählt werden. So ist es regelmäßig zu beobachten, dass Romane, die von der Literaturkritik einhellig missbilligt werden, dennoch die Bestsellerlisten wochenlang anführen. Blockbuster-Filme zählen in der Regel nicht zu den Werken, die mit den Preisen der Kritikerfestivals ausgezeichnet werden. Dennoch erzielen sie an den Kinokassen die meisten Kartenverkäufe.

Für dieses Problem bietet sich das Erklärungsmodell nach Adler (1985) an. Es lässt explizit von der Annahme ab, Qualität sei für Konsumenten vollständig informativ verfügbar, objektiv gegeben oder im Konsens durch alle Akteure anerkannt. Im Gegenteil, so Adler, sei Erfolg gerade wegen der unklaren Qualitätszuordnungen möglich. Der Grund liegt darin, dass für Käufer hohe Lern- und Suchkosten entstehen, wenn Informationen zur Qualität unklar sind. Zudem erhöht sich der Lern- und Suchaufwand, wenn der Konsumnutzen eines künstlerischen Produktes mit der Erfahrung des Konsumenten über das Genre und das künstlerische Feld steigt. Um diese Kosten letztlich gering zu halten, richten rationale Konsumenten ihre Kaufentscheidung auf genau jene Genres oder Künstler, die bereits die größte Popularität besitzen. Sie passen ihre Kaufentscheidung damit den Präferenzen anderer an und kaufen die Angebote, deren Nachfrage bereits hoch ist. Dies lässt sich dann diffusionstheoretisch dergestalt fassen, dass durch herdenartige Ansteckungsprozesse und Informationskaskaden die Nachfrage bis ins Unermessliche gesteigert wird, sobald eine ausreichende Menge anderer Akteure das Gut bereits nach- 
fragt oder für qualitativ hochwertig einschätzt und dies meinungsbildend kommuniziert (Banerjee 1992; Bikhchandani et al. 1992; Granovetter 1978; Keuschnigg 2012b; Lutter 2010; Rossman 2012).

Zusammenfassend lässt sich festhalten, dass sowohl Rosen als auch Adler sowie diffusionstheoretische Erweiterungen vor allem auf die Konsumentenseite bezogen sind und Erklärungen aus der Perspektive der Nachfrageseite bieten. Die Mechanismen auf dem Arbeitsmarkt der Künstler selbst sind daher bislang nicht einbezogen worden. Die Anbieterseite wird insofern berücksichtigt, als technische Entwicklungen die Voraussetzungen für extreme Nachfragekonzentration schaffen, nicht aber als direkte Erklärungen selbst herhalten. Vor allem liefern diese Ansätze keine Erklärung über die anfängliche Entstehung von Erfolg. Die Erklärung nach Adler setzt erst dann ein, wenn bereits eine hinreichende Masse an Nachfragern ihre Konsumentscheidung angeglichen hat. Diffusionstheoretische Ansätze modellieren zwar den Ausbreitungsverlauf, liefern aber keine Erklärung dafür, wann und wodurch kritische Werte erreicht werden, die dann Nachfragekonzentrationen erzeugen. So kann nach den Kaskadenmodellen von Bikhchandani et al. (1992) und weiteren diffusionstheoretischen Ansätzen zwar recht früh im Ausbreitungsprozess ein Überhang entstehen, der die Nachfrage konvergieren lässt. Welche Signale letztlich diesen Überhang auslösen, bleibt den Ansätzen exogen. Es fehlen damit die theoretischen Mechanismen, die die anfänglichen Erfolgsunterschiede bedingen und dann Potenzial für Erfolgskonzentrationen freisetzen. Im Folgenden soll daher die Anbieterseite betrachtet und der Frage nachgegangen werden, welche soziologischen Perspektiven die Entstehungsbedingungen für Erfolg und damit die Voraussetzungen für Konzentrationsprozesse in der Kreativbranche zu erklären imstande sind.

\section{Die Entstehung von Erfolg: Soziologische Ansätze}

\subsection{Askriptive Ungleichheiten auf Kreativarbeitsmärkten}

Bei dem Winner-take-all-Phänomen handelt es sich insbesondere deshalb im Kern um ein Problem sozialer Ungleichheit, weil sich das Verhältnis von Leistung und Erfolg stark auseinanderbewegt hat. Superstarphänomene weisen eine hochgradige Entkopplung von Leistung und Erfolg auf. So ist es nicht ungewöhnlich, dass die Erfolgreichsten gut das Hundertfache vom Durchschnitt verdienen, diese Summe allerdings nicht durch hundertfach größeres Talent gerechtfertigt ist. Sighard Neckel und Kai Dröge, die sich mit dem Winner-take-all-Phänomen beschäftigt haben (Neckel 2001, 2008; Neckel und Dröge 2003), fassen es daher zu Recht unter dem Begriff der „Gelegenheitsökonomien“ und betonen, dass nicht Leistung über Markterfolg entscheidet, sondern non-meritokratische Faktoren wie günstige Gegebenheiten, Marktlage und ,zur richtigen Zeit am richtigen Ort" zu sein.

Es ist daher sinnvoll, zur Erklärung des Phänomens zunächst die soziologische Ungleichheitsforschung heranzuziehen. Ohne Frage zählt innerhalb dieser Forschung die Untersuchung der Ursachen und Auswirkungen askriptiver Mechanismen auf Lebensverläufe und Arbeitsmarktchancen zu den wichtigsten Gegenstandsbereichen. Neben Benachteiligungen ethnischer Minoritäten oder aufgrund des Alters oder der sozialen 
Herkunft ist insbesondere geschlechtsspezifische Ungleichheit ein zentrales Thema (England 2005; Pager und Shepherd 2008).

Petersen und Saporta (2004) liefern in ihrer grundlegenden Analyse einen ordnenden Überblick zum gegenwärtigen Forschungsstand und verweisen insbesondere auf Mechanismen ,allokativer Diskriminierung“ als wesentliche Opportunitätsstruktur askriptiver Ungleichheitsprozesse auf Arbeitsmärkten. Diese Form ist besonders bedeutend auf Arbeitsmärkten in den Kreativbranchen (Lutter 2012a, S. 438). Angehörige benachteiligter Gruppen werden nach diesem Modell durch allokative Prozesse über Zeit von den relevanten Ressourcen zur Erzeugung von Erfolg systematisch ausgeschlossen. So führen Diskriminierungen, deren Existenz die Autoren als gegeben voraussetzen, bei Einstellungen, Beförderungen oder Kündigungen zur schrittweisen Verdrängung von Frauen oder Minoritäten aus den höheren Hierarchieebenen oder den erfolgreichen Positionen. Stattdessen werden sie in Bereiche gedrängt, die dauerhaft geringer entlohnt sind oder geringere Erfolgsmöglichkeiten bieten.

In den Kreativindustrien ist diese Form zum einen wegen des Fehlens einklagbarer Gleichstellungsstandards verbreitet. Die Kreativbranchen sind in aller Regel keine internen, sondern extern strukturierte Arbeitsmärkte und bieten überwiegend zeitlich befristete Tätigkeiten, die in Projektteams realisiert werden. Der ,projektbasierten Polis“ (Boltanski und Chiapello 2003, S. 147 ff.) fehlen aber die in traditionellen Arbeitsmärkten etablierten Institutionen, die möglichen Benachteiligungen juristisch entgegenwirken können.

Zum anderen sorgt das sehr hohe Allokations- und Fluktuationsaufkommen dieser Märkte dafür, dass es strukturell viel mehr Gelegenheiten für Diskriminierungen gibt. Die Kreativbranche zeichnet sich durch ein sehr hohes Allokationsaufkommen aus, weil Projektteams ständig neu besetzt, zusammengestellt und aufgelöst werden müssen, Faulkner und Anderson fassen diesen Zwang zur Dauererneuerung im Begriff der ,adhocracy“ (Faulkner und Anderson 1987, S. 880). Engagements für Schauspieler dauern in aller Regel zwischen wenigen Tagen und mehreren Wochen, länger zumeist nicht. Nach Abschluss der Dreharbeiten widmet sich das Ensemble neuen Projekten; jeder geht wieder seinen Weg.

Mit dieser Flexibilität geht ein hoher Grad an Informalität bei der Rekrutierung von Personal einher (Eikhof und Haunschild 2007, S. 528). Es besteht dann die Gefahr, dass askriptive Merkmale und persönliche Beziehungen statt leistungsgerechte Beurteilungen relevant werden (vgl. bereits: Tilly 1998). Sind wichtigen Entscheidungsträger dazu überwiegend männlich, so können sich informelle Strukturen im Auswahlprozess negativ für Frauen auswirken. Die informelle Rekrutierungskultur der Kreativbranche gäbe somit strukturell größere Möglichkeiten zur systematischen geschlechts- oder minoritätenspezifischen Benachteiligung. Tatsächlich belegen Studien, dass askriptive Mechanismen sozialer Ungleichheit auf projektbasierten Arbeitsmärkten eine Rolle spielen. Filmproduzenten und Regisseure sind in der Filmbranche die wesentlichen Entscheidungsträger und sie sind häufig männlich (Levy 1989, S. 36). Ähnliches konstatiert Apitzsch (Apitzsch 2010, S. 73 f.) für den Bereich Kamera. Drehbuchautoren sind fast zu 90\% männlich (Bielby und Bielby 1996, S. 254). Die gesamte Branche hat einen ausgeprägten Mittelschichtsbias; Menschen mit Migrationshintergrund sind deutlich unterrepräsentiert (Grugulis und Stoyanova 2012, S. 1319). 
Bielby und Bielby (1996) zeigen, dass weibliche Drehbuchautoren über ihre gesamte Karriere hinweg kontinuierlichen Benachteiligungen ausgesetzt sind und im Vergleich zu männlichen Kollegen deutlich geringere Erfolgschancen aufweisen. Analog bestätigt sich für Filmschauspieler, dass Frauen, bei Gleichhaltung verschiedener Humankapitalfaktoren wie Berufserfahrung und Erfolg, eine signifikant geringere Überlebenschance haben als männliche Darsteller (Lutter 2012a). Entsprechend verdienen sie deutlich weniger (vgl. Bührmann et al. 2013, S. 15).

Lincoln und Allen (2004) argumentieren in ihrer Studie, dass geschlechtsspezifische Ungleichheit mit weiteren askriptiven Faktoren eine Negativspirale in Gang setzen kann, die dann Benachteiligungen insgesamt verstärkt. Die Studie weist für die Filmbranche Interaktionen zwischen alters- und geschlechtsspezifischen Diskriminierungen nach und zeigt, dass sowohl Frauen als auch ältere Akteure Benachteiligungen in ihren Chancen auf Erfolg erfahren, die Benachteiligungen jedoch besonders hoch sind, wenn beide Merkmale zusammentreffen. Die Erfolgsunterschiede von Frauen verstärken sich somit im Alter, sodass die älteren Frauen die geringsten Chancen auf Erfolg haben und strukturelle Benachteiligungen erfahren. Daraus ergibt sich Hypothese $H 1$ :

H1: Allokative Diskriminierungsprozesse führen zur systematischen Benachteiligung von Frauen und Angehörigen von Minoritäten und reduzieren die Chancen auf Erfolg, während nicht benachteiligte Gruppen dadurch höhere Chancen auf Erfolg besitzen.

Allerdings lassen sich Beispiele finden, in denen Frauen mit zunehmender Karrieredauer immer größeren Erfolg ansammeln. So belegt die oben angeführte Studie zu den Karrieren von Filmschauspielern (Lutter 2012a), dass Benachteiligungen zwischen Geschlechtern sich auf ein statistisch nicht mehr von Null zu unterscheidendes Niveau reduzieren, sofern Frauen in der Lage sind, sichtbare Erfolge in Form von Filmpreisen zu sammeln. Wenn also strukturelle Benachteiligungen durch diskriminierende Auswahlprozesse über andere Mechanismen ausgeglichen werden können, so muss die Erklärung ungleichen Erfolgs durch weitere Ansätze angereichert werden.

\subsection{Kumulierte Vorteile oder „Matthäus-Effekte“}

Ein solcher Ansatz aus der soziologischen Ungleichheitsforschung ist die ,,Theorie kumulierter Vorteile“ (DiPrete und Eirich 2006), welche einen Mechanismus beschreibt, mit dem es möglich wird, das Zustandekommen extremer Erfolgsverteilungen zu erklären. Der Ansatz ist als „Matthäus-Effekt“ bekannt und geht auf Robert K. Mertons Essay über den Erfolg von Wissenschaftlern und die Rolle von Zitationshäufigkeiten und Reputation zurück (Merton 1968; Neckel et al. 2010, S. 448 ff.; Zuckerman 2010). ${ }^{6}$

In einer klassischen empirischen Studie zu diesem Thema zeigen Allison et al. (1982, S. 617), dass Publikationserfolge unter Wissenschaftlern im Karriereverlauf immer ungleicher werden. Bei jungen Kohorten bestehen noch keine großen Unterschiede, aber

6 Der Name versteht sich als Anlehnung an das Gleichnis von den anvertrauten Talenten aus dem Matthäus-Evangelium: „Denn wer da hat, dem wird gegeben, dass er die Fülle habe; wer aber nicht hat, von dem wird auch das genommen, was er hat" (Matthäus Evangelium 13:12). 
mit steigender Berufserfahrung konzentriert sich der Erfolg zunehmend. Ältere Kohorten zeichnen sich dann durch große Unterschiede aus. Während die Gesamtmasse der etablierten Wissenschaftler relativ wenige Publikationserfolge aufweist, ist nur eine kleine Anzahl der Wissenschaftler sehr erfolgreich.

Die Theorie kumulierter Vorteile nimmt an, dass gegenwärtige Erfolge durchschnittlich häufiger nicht durch aktuelle Leistungen, sondern durch früher bereits erlangte Gewinne erzielt werden (vgl. Lutter 2012a, S. 439). Der Grund liegt darin, dass Erfolg Aufmerksamkeit hervorruft, die wiederum neue Ressourcen ermöglicht, womit das Erzielen weiterer Erfolge wahrscheinlich wird. So werden bei Wissenschaftlern Publikationserfolge durch bessere Laborausstattung, mehr Mitarbeiter, weniger Lehre, mehr Forschungsfreisemester etc. belohnt, womit weitere Erfolge ermöglicht werden. Durch diese Rückkopplungseffekte stellt zukünftiger Erfolg eine Funktion des früher bereits erlangten dar.

Unter den Bedingungen dieses Verlaufs nehmen die Erfolgsunterschiede zwischen Akteuren extreme Ausmaße an. Aus kleinen anfänglichen Vorteilen können im Zeitverlauf sehr große Unterschiede heranwachsen, obwohl sich Akteure in ihrem Talent, ihrer Produktivität oder Qualifizierung wenig unterscheiden (Lynn et al. 2009). Die Anerkennungen, die den erfolgreichen Akteuren entgegengebracht werden, verstärken die Ungleichheit zusätzlich, weil Anerkennungen der erfolgreichen Akteure eine höhere soziale Akzeptanz und Aufmerksamkeit erfahren und damit den Erfolgreichen weiteren Status verleihen (Gould 2002, S. 1146). Der Matthäus-Mechanismus perpetuiert sich damit nicht nur auf der Ebene der Ressourcen- und Machtausstattung, sondern durch soziale Zuschreibungsprozesse auch auf der Ebene der Aufmerksamkeit. ${ }^{7}$ Während für die Erfolgreichen damit soziale Aufwärtsspiralen weiterer Anerkennung entstehen, stagniert die Zahl der Erfolge für die weniger Erfolgreichen, die ohne neue Erfolge keine weiteren erzielen können. Leistung und Erfolg driften infolgedessen auseinander. Es ergeben sich soziale Schließungsprozesse, die die Gewinne stabil auf die wenigen erfolgreichen Akteure konzentrieren.

Auch hier wieder lässt sich annehmen, dass diese Mechanismen besonders virulent sind auf Arbeitsmärkten, in denen permanente „Kraftproben“ (Boltanski und Chiapello 2003, S. 361) Reputationsordnungen entstehen lassen, die Exklusivität und Qualität konstruieren und als Realität sui generis einen wesentlichen Einfluss auf Erfolg nehmen. So generiert sich in der Wissenschaft Reputation aus mit dem richtigen Impact-Faktor versehenen Publikationserfolgen und Zitationshäufigkeiten (Münch 2010); in der Filmbranche aus erfolgreich abgeschlossenen Filmprojekten und der Akkumulation von Filmpreisen (Lutter 2012a); in der Werbe- und Medienbranche über Ideenwettbewerbe und Preisvergaben (Koppetsch 2006; 2008). Allgemein lässt sich aus dieser Diskussion daher die folgende Hypothese aufstellen:

H2: Früherer Erfolg bestimmt zukünftigen Erfolg. Akteure, die frühzeitig mehr Erfolg für sich akkumulieren können, haben unabhängig von ihrer aktuellen Leistung und Produktivität größere Aussicht auf zukünftige Erfolge.

7 Vgl. Bothner et al. (2010) für eine Studie zur mathematischen Modellierung. Zum Zusammenhang von Anerkennung und Erfolg, empirisch untersucht anhand der Rezeptionshäufigkeit preisgekrönter sozialwissenschaftlicher Aufsätze, vgl. Diekmann et al. (2012). Zur enormen Stabilität dieser Winner-take-all- Hierarchien, vgl. Van de Rijt et al. (2013). 


\subsection{Netzwerkeinbettung und die Stärke schwacher Beziehungen}

Ein dritter soziologischer Ansatz zur Erklärung von Winner-take-all-Verteilungen lässt sich aus der sozialen Netzwerkforschung ableiten. Hier stellt insbesondere das von Mark Granovetter geprägte Konzept von der „Stärke schwacher Beziehungen“ (Granovetter 1973, 1974) sowie die Weiterführungen durch Ronald Burt im Konzept des „structural hole“ (Burt 1992) ein Modell zur Erklärung von Erfolgsungleichheiten dar. Das Konzept besagt, dass jene Akteure, die über ein breites Netzwerk loser, schwacher oder unregelmäßiger Beziehungen verfügen, bessere Ressourcenzugänge und damit potenziell größeren Erfolg haben, als Akteure, die in dichten Netzwerken mit regelmäßigen Kontakten eingebunden sind.

Der Grund liegt darin, dass Akteure mit vielen schwachen Kontakten über einen breiteren Informationspool verfügen und einer größeren Variationsvielfalt unterschiedlichster Anschauungen, Traditionen, Ressourcen und Kontakten ausgesetzt sind, die für die Akquise neuer Projekte und Tätigkeiten genutzt werden können. Akteure in engen Netzen haben dagegen einen eingeschränkten Diversifikationsgrad.

Die Erfolg mindernde Eigenschaft dichter Netzwerkeinbettung verschärft sich durch mehrere Aspekte. Zum einen monotonisieren Tendenzen sozialer Homophilie den ohnehin eingeschränkten Informations- und Ressourcenpool weiter. Soziale Homophilie ist die in der Netzwerkforschung thematisierte Eigenschaft, dass sich Personen, die einander bekannt sind und in häufigem Kontakt zueinander stehen, in ihren Meinungen, Einstellungen und im Verhalten angleichen und jedem einzelnen Mitglied ein geringeres Maß sozialer Abweichung gewähren (McPherson et al. 2001). Enge Netze sind durchschnittlich häufiger homogen und haben einen eingeschränkteren Zugang zu alternativen Meinungen, sanktionieren abweichende Meinungen stärker durch ein höheres Maß sozialer Kontrolle und sind folglich devianten Verhaltensweisen weniger häufig ausgesetzt.

Versiegen die Ressourcen, sind Angehörige kohäsiver Kreise weniger gut abgesichert. Denn bricht ein Teil des Netzes weg, dann bleiben nicht viele Alternativverbindungen, die für den Ressourcenzugang genutzt werden können. Für Akteure mit vielen, aber zwangsläufig schwächeren Beziehungen stellen im Fall des Wegbrechens genügend weitere Netze die Versorgung mit überlebenswichtigen und Erfolg bestimmenden Informationen sicher, mit denen neue Ressourcen und damit Überleben und Erfolg gesichert werden können. Die Ausbreitung des eigenen Netzwerkes bewirkt also die Diversifikation des Risikos; je breiter das Netzwerk, desto geringer das Ausfallrisiko.

Gerade für projektförmig organisierte Arbeit hat die Forschung auf informelle Rekrutierungspraktiken und die Rolle von Netzwerken hingewiesen (Eikhoff und Haunschlid 2007; Gottschall 1999; Grugulis und Stoyanova 2012; Henninger und Gottschall 2007; Jones 1996; Mathieu 2012; McKinlay und Smith 2009). Das „unternehmerische Selbst“ (Bröckling 2007), so eine Schlussfolgerung dieser Forschung, ist auf Netzwerkvielfalt angewiesen. Mehrere empirische Studien haben diese Zusammenhänge quantitativ untersucht. Giuffre (1999) erfasst die professionellen Netzwerke zeitgenössischer Fotografen in New York über einen Zeitraum von elf Jahren und zeigt, dass jene den größten Erfolg entwickeln, die in größere Netzwerke mit losen Bindungen zu Galerien und Kunstvermittlern eingebunden sind. Diejenigen dagegen, die wiederkehrend mit den gleichen Galeristen zusammenarbeiten, die also in wenige engmaschige Netze einge- 
bunden sind, weisen deutlich geringere Erfolgschancen und prekäre Daseinsbedingungen auf.

Die Studie belegt damit empirisch, dass Künstlerkarrieren weniger vom individuellen Talent per se abhängig sind, sondern von der strukturellen Position im sozialen Gefüge. Wenn aber individuelle Karriereerfolge von der strukturellen Netzwerkposition abhängen, dann besagt dies, dass jede Änderung im Netzwerk auch veränderte Karrierebedingungen für jeden Einzelnen hervorruft. Fallen zentrale Knoten weg, so wandelt dies die Struktur des Netzes dahingehend, dass das Beziehungsgeflecht aller Akteure betroffen oder berührt wird. Karriere ist dann mit Giuffre (1999) nicht atomistisch, als linear verlaufende „Karriereleiter“ zu verstehen, sondern vielmehr als „Sandhaufen“, auf dem der Karriereverlauf eines jeden Akteurs Veränderungen im Gesamtsystem verursacht, die wiederum Einfluss auf die Karrierechancen jedes einzelnen nehmen können. Erfolg hängt damit mehr von den sozialen Strukturen des gesamten Gewebes ab als von den individuellen Talenten (siehe dazu ähnlich für den Karriereerfolg von Malern: Accominotti 2009).

Eine weitere paradigmatische Studie findet sich bei Uzzi und Spiro (2005), die die Künstler-Teams von Broadway-Musicals im Hinblick auf ihre Zusammensetzung und ihren Erfolg untersuchen. Zentrales Ergebnis ist, dass der künstlerische Erfolg der Produktion eine umgekehrt u-förmige Funktion der Netzwerkdichte des Teams darstellt. Gemessen an der Anzahl früherer Gelegenheiten zur Zusammenarbeit zwischen Teammitgliedern steigen zunächst die Erfolgschancen auf ein Maximum an, um dann rapide abzusinken. Bei zu hoher Einbettung sinken die Vorteile sozialen Kapitals; positive Wirkungen kehren sich ins Gegenteil. Dies stellt eine indirekte Bestätigung der These Granovetters dar: Um Erfolg zu erzielen, sind Akteure zwar einerseits auf den Informationsfluss durch frühere Kontakte angewiesen, jede Über-Einbettung aber verhindert die Chancen auf Erfolg.

Ähnlich zeigt Rost (2011), dass der mittlere Einbettungsgrad den besten Einfluss auf kreative Innovationsfähigkeit hat. Teams benötigen eine relevante Mindestanzahl an starken Beziehungen und wiederholten Kollaborationen, zugleich eine hinreichende Menge schwacher Beziehungen. Nur dann, so das Argument der Studie, kann sich die positive, informationsfördernde Kraft schwacher Beziehungen überhaupt entfalten, weil starke Beziehungen ausreichend Stabilität vermitteln. Sofern also keine Unter- oder ÜberEinbettung vorliegt, wirken beide Pole im Kreativitätsfluss komplementär zueinander und bestärken sich gegenseitig in ihren positiven Eigenschaften (vgl. auch: Cattani und Ferriani 2008; Ferriani et al. 2009). Daraus ergibt sich die folgende Annahme:

H3: Bei hinreichender Zahl schwacher Beziehungen wachsen die Chancen auf Erfolg mit Zunahme kohäsiver Netzwerke auf ein Maximum und sinken dann mit jeder weiteren Steigerung in der Dichte der Einbettung.

\subsection{Erfolg als Rollenausdehnung}

Ein vierter Ansatz bezieht marktsoziologische Forschung aus dem Bereich der Organisationsökologie ein (vgl. grundlegend: Hannan und Freeman 1977), die sich in zahlreichen Studien mit den Auswirkungen sozialer Positionen, Rollen- und Kategorienzugehörig- 
keiten auf Marktordnungen, Gründungs- und Mortalitätsraten sowie Markterfolg in Populationen unternehmerischer Organisationen oder Individuen beschäftigt. Ein gut bestätigter Befund dieser Forschungsrichtung ist, dass Generalisten, also Akteure mit breiter Nischenausdehnung, Marktnachteile erfahren, während Akteure mit geringer Ausdehnung und fokussierter Identität (Spezialisten) bessere Chancen auf Erfolg und Überleben am Markt haben (Carroll et al. 2002; Carroll und Swaminathan 2000; Dobrev et al. 2001; Hsu 2006; Hsu et al. 2009).

Erstens liegt ein Grund darin, dass mit steigender Ausdehnung der Durchdringungsgrad jeder einzelnen angesprochenen Kategorie notwendigerweise beschränkt sein muss. Jedes Genre hat unterschiedliche Anforderungen, Kulturtechniken, Traditionen und soziale Anhängerschaften, deren Ziele und Erwartungen über verschiedene Genres sogar entgegengesetzt sein können. Der Erwartungs- und Anforderungsdruck ist bei hoher Ausdehnung damit hoch.

Zweitens ist die Legitimität beim Publikum gering, weil keine klaren Zuordnungen erkennbar sind. Marktakteure mit breiter Ausdehnung haben gegenüber Spezialisten den Nachteil, dass sie jede einzelne Kategorie nicht mit der gleichen Intensität und Ressourcenausstattung nutzen können. Damit bieten sie tendenziell geringere Qualität und Service an. Ein Musiker, der zugleich Klassik, Popmusik und Jazz produziert, wird in jedem einzelnen Genre weniger Legitimität und Erfolg erfahren, weil zwangsläufig nicht jede Kategorie mit der vollen Ausstattung und Energie bearbeitet werden kann. Zudem entstehen beim Genrepublikum Ungewissheiten und Zweifel über Qualität oder Eignung.

Ein dritter Grund liegt darin, dass mit größerer Ausdehnung eine verschlechterte Position im Wettbewerb herrscht, weil Generalisten in jeder einzelnen Kategorie durch Konkurrenten mit besserer Durchdringung und Ressourcenallokation übervorteilt werden können. Mit jeder weiteren abgedeckten Kategorie nimmt die Zahl der Konkurrenten insgesamt zu - und die Erfolgswahrscheinlichkeit ab.

Spezialisten hingegen beschränken sich auf eine Kategorie, füllen diese aber mit größter Intensität aus. Sie können sämtliche Ressourcen dem Ausbau dieser Nische zuwenden. Hierdurch stoßen sie bei den Nachfragern auf größere Legitimität und schaffen eine Identität, die ihren Erfolg steigern kann. Ebenso sind sie gegenüber Konkurrenten besser geschützt, da sie über Informations- und Erfahrungsvorteile verfügen, ihren Markt „,kennen“, und aufgrund ihrer Spezialisierung bessere Qualität anbieten können. Sie durchdringen ihre Kategorie. Etablieren sie eine Marke, das heißt, füllen sie die Nische mit ihrem Produkt derart, dass die gesamte Nische ausschließlich mit dem Anbieter gleichgesetzt wird und das Markenprodukt zur „sozialen Tatsache“ reift (Hellmann 2003), dann kann die Marktnachfrage fast vollständig auf sich vereint, der Erfolg monopolisiert und maximale „Monopolrenten“ daraus gezogen werden.

Tatsächlich bestätigen Untersuchungen auf Kreativmärkten diese Zusammenhänge. So schließen Zuckermann et al. (2003) aus einer Studie über Filmkarrieren von Schauspielern, dass Filmakteure ihre Chancen auf Erfolg erhöhen, wenn sie ihre Karriere mit einer eng fokussierten Identität beginnen, also eine bestimmte Nische ausfüllen und sich darin einen Namen machen. Allerdings, so zeigen die Autoren, birgt das die Gefahr, sich mit der eng ausgerichteten Identität zu sehr auf eine Kategorie zu verlassen. Besonders in der volatilen Filmbranche, in der sich Moden, Geschmack und Kategorien sehr schnell ändern können, besteht die Gefahr, mit einem zu engen Fokus abgehängt zu werden. 
Die erfolgreichsten Akteure verlassen daher ihre enge Fokussierung und dehnen ihre Ausrichtung auf weitere Genres aus. Dies ist konsistent mit Dobrev et al. (2001), die betonen, dass auf Märkten mit (noch) unklaren oder schnelllebigen Kategoriensystemen eine breite Kategorienausdehnung insofern vorteilhaft sein kann, als sie die Anpassungschancen in sich rasant verändernden Umwelten erhöht. Spezialisten können also dort Nachteile haben, wo Märkte wechselhafte Kategoriensysteme aufweisen oder diese sich erst noch ausbilden müssen.

Um aber überhaupt erst Reputation aufzubauen, müssen Akteure zunächst mit einer fokussierten Identität auf hinreichend etablierten Märkten eine Nische besetzen, bevor sie ihr Spektrum, ohne Erfolgsverluste hinnehmen zu müssen, erweitern können. Park und Podolny (2000) argumentieren, dass die Nischenausdehnung insbesondere dann zu Erfolg führt, wenn Marktakteure in ihrem Kerngeschäft einen hohen Status etablieren. Anhand von Investmentbanken zeigen die Autoren, dass jene Banken ihren Erfolg maximieren, die zuerst in einer Nische über hohe Reputationsvorteile verfügen und dann auf weitere Nischen expandieren. Ähnlich zeigen Rao et al. (Rao et al. 2003, S. 839) für die französische Gastronomie, dass Restaurantchefs mit größter Reputation in klassischer französischer Küche ihren Erfolg dann steigern, wenn sie Elemente der neu aufgekommenen nouvelle cuisine aufnehmen, also ihre Kategorienabdeckung rechtzeitig ausdehnen. ${ }^{8}$

Marktakteure werden also dann mit größtem Erfolg belohnt, wenn sie zuerst ihre Reputation innerhalb einer fokussierten Spezialisierung aufbauen, um Legitimität beim Publikum zu erzeugen, um dann ihre Kategorienabdeckung zu erweitern, womit weiterer Erfolg aufgebaut wird.

H4: Auf hinreichend etablierten Märkten haben Akteure dann größte Chancen auf Erfolg, wenn sie zunächst innerhalb einer fokussierten Nische Status aufbauen und anschließend ihre Nische ausdehnen und erweitern.

\subsection{Heterarchie oder: Organisierte Dissonanz}

Der fünfte Erklärungsansatz stellt eine Verbindung von Netzwerk- und Nischentheorie dar und geht im Wesentlichen auf die Arbeiten von David Stark und Kollegen zurück (Beunza und Stark 2004; Girard und Stark 2002; Stark 2009; De Vaan et al. 2011; Vedres und Stark 2010). Stark untersucht die Bedingungen für Innovationen und Erfolg in kreativen Gruppenprozessen. Dies geschieht teilweise mit ethnografischen, teilweise mit quantitativen Methoden und in verschiedenen empirischen Kontexten wie New-MediaFirmen in New York's Sillicon Alley, Börsenhändlern im New York der 2000er-Jahre oder Produktionsteams von Computer- und Videospielen.

Destillat seiner Forschungen ist das theoretische Konzept der „organisationalen Heterarchie“, mit dessen Hilfe eine soziologische Erklärung für den Erfolg von Innovationen möglich wird. Hiernach stellt die spezifisch heterogene Organisation von Kreativität eine wesentliche und entscheidende Quelle für die Innovationsfähigkeit und den Erfolg krea-

8 Weitere Beispiele aus der Popmusik ließen sich aufführen, so etwa das betonte Bestreben deutscher Gangsta-Rapper, die von ihnen besetzte und selbst erschaffene Nische zu verlassen (Seeliger 2012, S. 77) 
tiver Gruppen dar. Ihr wesentliches Element ist die organisierte Herstellung von Dissonanz. Starks Studien zeigen, dass Teams, die aus unterschiedlichen kreativen Bereichen zusammengestellt und an der Schaffung neuer Produkte beteiligt werden, höhere Aussichten auf Innovationserfolg haben. Werden Teammitglieder aus verschiedenen kreativen Bereichen rekrutiert, dann hat die auf diese Weise „organisierte“ Dissonanz den Effekt, dass bei der Suche nach Innovationen permanente Konflikte entstehen, die den multiplen Bewertungsstandards und unterschiedlichen ,Schulen“ oder Erfahrungshintergründen der zusammengebrachten Akteure geschuldet sind. Durch diesen Reibungsprozess ergeben sich Unsicherheiten darüber, welche Maßstäbe in welcher Art auf die zu erschaffende Innovation angesetzt werden sollen. Hierdurch werden Akteure gezwungen, bis dato als unhinterfragt geltende Maßstäbe in einem neuen Licht zu betrachten. Manche Standards müssen so völlig neu ausgehandelt oder begründet werden. Auf diese Weise eröffnen die zusammengebrachten Dissonanzen einen Unsicherheitsraum, der es den Akteuren ermöglicht, bekannte Pfade zu verlassen und kreative Leistungen zu erbringen.

Die Heterogenität der kreativen Köpfe schöpft damit den Raum für die Suche nach innovativen Lösungen optimal aus. Stark zeigt gemeinsam mit Daniel Beunza in einer Studie über Börsenhändler (Beunza und Stark 2004), wie Techniken der Dissonanzschaffung Gewohnheiten durchbrechen und als Folge kreative Innovationen hervorbringen. Eine solche Technik ist die zufällige Rotation der Sitzordnungen der Mitarbeiter in einem Büro. Durch die Veränderung der Sitzordnungen werden Gewohnheiten regelmäßig zerstört und der personelle Austausch der Kollegen unter einander maximiert. Mit dem Austausch von Mitarbeitern werden auch neue Horizonte, Erfahrungen und Maßstäbe untereinander ausgetauscht (vgl. auch Beunza und Stark 2003, S. 139), die den internen Informationsfluss erhöhen. Die organisierte Dissonanz stellt somit den maximal möglichen Grad an Vielfalt bei gleichzeitiger Einheit her.

Eine weitere Studie zeigt, dass Unternehmen dann eine hohe Performanz aufweisen, wenn sie aus Gruppen bestehen, in denen ein Anteil ihrer Mitglieder zugleich mehreren externen Unternehmen angehört (Vedres und Stark 2010). Auch hierüber wird die Dissonanz durch die Verbindung unterschiedlicher Erfahrungen erhöht. In Analogie zu Ronald Burts netzwerktheoretischem Konzept des „structural hole“ nennen Vedres und Stark die multiple Verbindung mehrerer Netzwerke ,structural fold“, um das gleichzeitige Bestehen von Verbundenheit und Diversität zu unterstreichen. Im Unterschied zu Uzzi und Spiro (2005) argumentieren Vedreß und Stark auf einer weiteren Ebene: Nicht die kurvilineare Netzwerkkohäsion vorheriger Beziehungsaktivitäten entscheidet über den Erfolg in kreativen Teams, sondern die Existenz von Akteuren, die zugleich Mitglied in verschiedenen Teams aus unterschiedlichen Genres sind. Erst durch diesen gleichzeitigen Zusammenschluss von Heterogenität auf der einen und der nötigen Familiarität und Vertrauen auf der anderen Seite werden die Chancen auf erfolgversprechende Innovationsleistungen erhöht (vgl. zu Videospielproduktionen: De Vaan et al. 2012). ${ }^{9}$

9 Auch in der Managementliteratur finden sich Studien mit ganz ähnlichen Schlussfolgerungen. So zeigen Perretti und Negro (2007) anhand der Zusammenstellung von Filmteams, dass ein höherer Anteil an relativ unerfahrenen Neulingen die Innovationsfähigkeit, gemessen an der Etablierung neuer Genrekombinationen, positiv beeinflusst. 
Hieraus ergibt sich die Annahme, dass diejenigen Gruppen den höchsten Innovationsgrad aufweisen und damit bei den Kritikern des Kunstfeldes auf die größte Anerkennung stoßen, die kreative Dissonanzen bestmöglich in ihrer Gruppe miteinander verbinden. Diese besondere Governancestruktur der organisierten Dissonanz müsste deswegen den größten Erfolg auf sich vereinen können, weil Kritiker des eigenen Kunstfeldes in der Regel diejenigen Produkte mit der größten Anerkennung und „praise value“ (Hutter 2011) versehen, die aus Sicht der Experten- und Kritikerhierarchie die größte Innovationskraft im Feld haben (Allen und Lincoln 2004). Es ergibt sich demgemäß die folgende Hypothese:

\section{H5: Akteure in heterarchisch organisierten kreativen Gruppen haben größte Chancen auf Erfolg.}

\subsection{Status-Matching oder: Netzwerkspiralen des Erfolgs}

Ein letzter, und vielleicht der wichtigste dezidiert soziologische Mechanismus zur Erklärung von Erfolg auf Winner-take-all-Märkten ist die Tendenz vieler Künstlerarbeitsmärkte, die Zusammenstellung von Projektteams hochgradig nach Status zu ordnen. Ganz ähnlich zu den Mechanismen, die aus der soziologischen Ungleichheitsforschung für bildungshomogame Eheschließungen bekannt sind (Blossfeld 2009; Blossfeld und Timm 1997; Skopek et al. 2011), finden auch auf Künstlerarbeitsmärkten statushomogame Matching-Prozesse bei der Rekrutierung von Projektteams oder bei Kollaborationen zwischen Künstlern und Vermittlern statt.

Status ergibt sich aus mehreren Dimensionen, einmal aus den zugeschriebenen Urteilen und Rangordnungen der Akteure und Experten im Markt, dann aus dem Status derjenigen, die die Urteile abgeben sowie aus den Statusordnungen der Personen, mit denen Akteure zusammenarbeiten oder in der Vergangenheit zusammengearbeitet haben (Podolny 2001, 2005). Weil dies einen Einfluss auf die eigene Statusbeurteilung nehmen kann, besteht die Tendenz, mit anderen Akteuren des gleichen oder höherrangigen Status zu kollaborieren. Projektteams ,verklumpen“ so in nach Erfolg oder Status strukturierte Netzwerke. Es entstehen „Statusmärkte“ (Aspers 2007, S. 435 f.) mit festen Verbindungen von sehr erfolgreichen, mittelmäßig erfolgreichen und vielen wenig erfolgreichen Akteuren. Diese „Netzwerke des Erfolgs“" generieren dann unter den Bedingungen des Matthäus-Effekts weiteren Erfolg für ihre Angehörigen. Entsprechend stellt eine Studie über die Bestimmungsgrößen der Nominierung für einen Oscar heraus (Rossman et al. 2010, S. 46), dass nicht nur leistungsrelevante Aspekte zählen, etwa Berufserfahrung oder das Budget der Produktion, sondern auch die Reputation der Crew. Faulkner und Anderson (1987) zeigen anhand umfassender Kollaborationsdaten aus der Filmbranche, dass sich Teams hochgradig nach Status ordnen. Akteure aber, die es nicht schaffen, in diese Netze hineinzugelangen, fallen langfristig heraus und werden vom Erfolg abgehängt.

Die Statushomogamien auf dem Arbeitsmarkt für Filmschaffende werden nach Faulkner und Anderson (1987) im Wesentlichen durch zwei Bedingungen forciert: die Organisation der Arbeit in flexiblen Projektteams sowie die Unsicherheiten im Hinblick auf Qualität und voraussagbaren Erfolg und die Rolle von Reputation. Zum einen besteht ein Zwang zur permanenten Neurekrutierung der Teams. Damit ist das Fluktuationsaufkommen in projektbasierten Branchen ungleich höher als in klassischen Arbeitszusammen- 
hängen. Teams formieren sich ständig neu; Angebot und Nachfrage treffen kontinuierlich aufeinander. Zum anderen operieren die Teams in einem Umfeld hochgradiger Unsicherheit. Jedes künstlerische Produkt ist einzigartig und sein Erfolg schwer vorherzusagen. Es gibt Ungewissheiten in Bezug auf die Kosten, vorgefasste Konditionen können sich im Laufe des Projekts ändern, Kapital- und Finanzierungsströme unvorhergesehen variieren. Arbeitsabläufe und Entscheidungsprozesse sind wenig standardisiert; es gibt keine Routine. Viele Prozesse sind durch Experimentieren geprägt, durch Ausprobieren und Spontanität. Direktiven, Ziele und Vereinbarungen werden oft am Set neu ausgelegt, geändert, angepasst. Jedes Mitglied im Team muss imstande sein, darauf flexibel, kreativ und vielseitig zu reagieren. Der gegenseitige Interpretations- und Abstimmungsbedarf ist enorm hoch; es bestehen Probleme „doppelter Kontingenz“ (Luhmann).

Aufgrund dieser Unwägbarkeiten und des Fehlens objektiver Kriterien zur Beurteilung von Qualität müssen Akteure externe Signale heranziehen, die Auskunft über Qualität geben. Reputation, die aus früheren Projekten erworben wurde, spielt eine entscheidende Rolle bei der Beurteilung fachlicher Leistungen. Reputation muss jedoch durch frühere erfolgreiche Projekte erst hergestellt werden (Faulkner und Anderson 1987, S. 881). Je erfolgreicher die Beteiligung, desto mehr Reputation kann daraus gezogen werden. An Engagements mit hohem Prestige gelangen Akteure allerdings nur mit bereits bestehender guter Reputation. Dies stellt eine fast unüberwindbare Hürde dar, denn ,each credit in this business increases a person's chances for future work, and each money-earning production increases a person's chances for future contracting with colleagues who themselves are associated with successful ventures“ (Faulkner und Anderson 1987, S. 907).

Wie aber gelingt es Akteuren, diese Hürde zu nehmen? Wie können sie in statushöhere Teams gelangen, von deren Reputation sie profitieren können? Hier kann mit dem als „middle-status-conformity“ bekannten Ansatz (vgl. grundlegend: Phillips und Zuckerman 2001) argumentiert werden, dass Konformität und damit soziale Schließung besonders in Teams mit mittleren Statuslagen hoch ausgeprägt sind. Empirisch bestätigen dies etwa Perretti und Negro (2006). Am Beispiel von Filmteams zeigen sie, dass sowohl Teams mit hohem als auch geringem Status es häufiger wagen, Newcomer ohne Reputation zu integrieren. Der Grund dafür liegt zum einen darin, dass etablierte Akteure mit hoher Reputation in ihrem Zwang zur Neuheitenproduktion immer höhere Innovationsgrade erreichen müssen, um ihren etablierten Status zu verteidigen. Deswegen suchen sie permanent nach Wegen, konventionelle Ordnungen zu durchbrechen. Zum anderen erlaubt ihnen ihr etablierter Status wesentlich mehr Freiheitsgrade in der Richtungsgestaltung ihrer künstlerischen Outputs, ohne die Unterstützung einer breiteren Anhängerschaft zu verlieren. Ebenso bringt höherer Status entsprechend ökonomische Absicherung mit sich, die es erlaubt, etwaige Risiken einzugehen, die mit der Verpflichtung junger Talente oder der Etablierung neuer Genres verbunden sein könnten. So ist auch die geringe Durchlässigkeit in den mittleren Lagen zu erklären, die mit der entsprechend größeren ökonomischen Unsicherheit sowie der noch wenig etablierten Reputation zusammenhängt.

H6: Akteure haben dann die größte Chance auf Erfolg, wenn sie häufiger mit Teams zusammenarbeiten, deren Mitglieder einen hohen Status aufweisen und bereits erfolgreich sind. 


\section{Schluss}

Der Aufsatz hat das Potenzial soziologischer Ansätze zur Erklärung des Winner-take-all-Phänomens ausloten wollen. Auf Basis breiter Literatursichtung wurden sechs soziologisch-strukturelle Ansätze diskutiert, mit denen die Bedingungen von Erfolgsungleichheiten erklärt werden können. Der Beitrag versteht sich als eine soziologische Ergänzung der bestehenden dominanten Erklärungsansätze. Während bisherige Ansätze die Nachfrageseite betrachtet haben, stellt dieser Artikel Konditionen auf der Anbieterseite heraus. Das Hauptargument jedes der diskutierten Ansätze ist, dass Erfolgsbedingungen und -konzentrationen aus den sozialen Strukturen heraus erklärt werden müssen, in die die Akteure des Arbeitsmarktes eingebettet sind.

Ziel dieser Studie war auch, zukünftiger empirischer Forschung einen Überblick zum Forschungsstand und zu Möglichkeiten einer soziologisch-strukturellen Analyse des Winner-take-all-Phänomens zu eröffnen. Allerdings bleibt hervorzuheben, dass die Ansätze nicht geeignet sind, Erfolge für den Einzelfall vorherzusagen, zum Beispiel der fulminante Erfolg der Beatles oder der Aufstieg von Bill Gates (Gladwell 2008). Jeder der vorgestellten Ansätze beschreibt Konstellationen und Bedingungen, die im statistischen Mittel, alle Akteure zusammengenommen, die Chancen auf Erfolgskonzentrationen wahrscheinlicher machen. Zudem reicht jeder Ansatz für sich allein genommen nicht aus, um die Entstehung des Winner-take-all-Phänomens hinreichend zu erklären. Es handelt sich nicht um konkurrierende, sich gegenseitig ausschließende Thesen, sondern um einander ergänzende Ansätze. So können sich askriptive Ungleichheiten unter den Bedingungen des Matthäus-Effekts gegenseitig verstärken; auch Netzwerkkonstellationen können sich wechselseitig mit Matthäus-Effekten, Rollenausdehnungen oder askriptiven Prozessen beeinflussen und Erfolgskonzentrationen wachsen lassen. Mit anderen Worten: Jeder Ansatz kann miteinander multiplikativ interagieren. Allerdings ist a priori keineswegs klar, in welcher Richtung dies geschieht. Neben der Verstärkerwirkung können sich Ungleichheitstendenzen auch gegenseitig aufheben. Nur durch die theoretische und empirische Kombination und möglichst vollständige Betrachtung mehrerer Ansätze und ihrer potenziellen Interaktionseffekte gelangt man zu einem genaueren Verständnis der relevanten kausalen Prozesse. Dies kann selbstverständlich kein Beitrag alleine leisten: Es bleibt daher weiterer theoretischer wie empirischer Forschung überlassen, herauszufinden, welche Kombination dieser Ansätze in welchem Kontext letztlich die beste Erklärungskraft erzielt (vgl. erste quantitative Analysen: Lutter (2012a, 2013)).

Es war die Absicht des vorliegenden Beitrags, die weitere empirische Überprüfung durch zukünftige Forschung anzuregen. Denkbar wären viele Bereiche, auf die sich die dargestellten Mechanismen übertragen ließen. Im Vordergrund stehen vor allem konkrete Arbeitsmärkte in den Kultur- und Medienbranchen, etwa Erfolgsprozesse in den Karriereverläufen von Filmschaffenden, Erfolg und Marktbedingungen auf dem Buchmarkt, die Produktion von Musik und Formationsprozesse von Musikgruppen, der Erfolg von Musical-, Theater- oder Opernproduktionen. Über den rein künstlerischen Arbeitsmarkt hinaus wären Untersuchungsfelder in weiteren Bereichen denkbar, etwa die sozialstrukturellen Erfolgsbedingungen von Sportlern, die Erfolgsentwicklung von Architektenbüros, Karrieren und Kollaborationen von Wissenschaftlern oder Politikerlaufbahnen, Erfolg von 
Rechtsanwälten, Chirurgen oder Medizinern, Karrierebedingungen von Journalisten, Designern und Werbeleuten oder die Gehaltssteigerungen von Managern.

Eine ganz anders angesiedelte, aber indirekt relevante Untersuchungsfrage wäre, wie es zu erklären ist, dass Akteure ihre Berufswahl überhaupt auf diese Märkte verlegen. Warum wollen sie Schauspieler werden? Warum Musiker? Was sind ihre Motive? Die Beantwortung dieser Fragen würde zum einen eine mikrofundierte Erklärung auch für die Frage des Zustandekommens der Winner-take-all-Verteilungen bieten. Denn nur wenn Menschen über Motive verfügen, ihre Arbeitskraft in diesen Branchen zu investieren, können die Konzentrationsprozesse überhaupt entstehen. Nur wenn ein permanentes Überangebot besteht, können die Prekarisierungstendenzen und das Alles-oder-nichtsPrinzip weiter fortbestehen. Neben Selbstverwirklichung und bohemischem Lebensstil als Motivation (Eikhoff und Haunschild 2006), wirken hier möglicherweise die Erfolgskonzentrationen des Marktes selbst als Motivator, weil die exorbitanten Gewinne Träume und Fantasievorstellungen eines „Was wäre wenn“ anregen, ähnlich wie der Lottospieler vom großen Geldgewinn träumt und dies ihn trotz Verluste wöchentlich weiterspielen lässt (vgl. Beckert und Lutter 2007, 2008; Lutter 2012b). Zum anderen wäre diese Frage aus einer theoretischen Perspektive der Wirtschaftssoziologie interessant. Denn rationale, vollständig über ihre Chancen informierte Akteure würden Winner-take-all-Märkte meiden. Ein homo oeconomicus würde kein Schauspieler werden. Wie aber erklärt sich, dass es beständig mehr Personen in diese Berufe drängt, als es Nachfrage nach ihnen gibt?

Danksagung: Ich danke Birgit Apitzsch, Jens Beckert, Marc Keuschnigg, Renate Mayntz, Isabella Reichert, Frank Wehinger und den Herausgebern und anonymen Gutachtern der Kölner Zeitschrift für Soziologie und Sozialpsychologie für hilfreiche Kommentare zu einer früheren Version. Ich danke außerdem den Mitgliedern der Forschungsgruppe „Soziologie der Märkte“ am MPIfG für wertvolle Hinweise.

\section{Literatur}

Accominotti, Fabien. 2009. Creativity from interaction: Artistic movements and the creativity careers of modern painters. Poetics 37:267-294.

Adler, Moshe. 1985. Stardom and talent. American Economic Review 75:208-212.

Allen, Michael Patrick, und Anne E. Lincoln. 2004. Critical discourse and the cultural consecration of American films. Social Forces 82:871-893.

Allison, Paul D., J. Scott Long und Tad K. Krauze. 1982. Cumulative advantage and inequality in science. American Sociological Review 47:615-625.

Apitzsch, Birgit. 2010. Flexible Beschäftigung, neue Abhängigkeiten. Projektarbeitsmärkte und ihre Auswirkungen auf Lebensverläufe. Frankfurt a. M.: Campus.

Aspers, Patrik. 2007. Wissen und Bewertung auf Märkten. Berliner Journal für Soziologie 17:431-449.

Banerjee, Abhijit V. 1992. A simple model of herd behavior. Quarterly Journal of Economics 107:797-817.

Becker, Howard S. 1982. Art worlds. Berkeley: University of California Press.

Beckert, Jens. 2004. Unverdientes Vermögen. Soziologie des Erbrechts. Frankfurt a. M.: Campus. 
Beckert, Jens, und Jörg Rössel. 2004. Kunst und Preise: Reputation als Mechanismus der Reduktion von Ungewissheit am Kunstmarkt. Kölner Zeitschrift für Soziologie und Sozialpsychologie 56:32-50.

Beckert, Jens, und Mark Lutter. 2007. Wer spielt, hat schon verloren? Zur Erklärung des Nachfrageverhaltens auf dem Lottomarkt. Kölner Zeitschrift für Soziologie und Sozialpsychologie 59:240-270.

Beckert, Jens, und Mark Lutter. 2008. Wer spielt Lotto? Umverteilungswirkungen und sozialstrukturelle Inzidenz staatlicher Lotteriemärkte. Kölner Zeitschrift für Soziologie und Sozialpsychologie 60:233-264.

Beckert, Jens, und Patrik Aspers. Hrsg. 2011. The worth of goods: Valuation and pricing in the economy. Oxford: Oxford University Press.

Beckert, Jens, und Christine Musselin. Hrsg. 2013. Constructing quality: The classification of goods in markets. Oxford: Oxford University Press.

Beunza, Daniel, und David Stark. 2003. The organization of responsiveness: Innovation and recovery in the trading rooms of Lower Manhattan. Socio-Economic Review 1:135-164.

Beunza, Daniel, und David Stark. 2004. Tools of the trade: The socio-technology of arbitrage in a Wall Street trading room. Industrial and Corporate Change 13:369-400.

Bielby, Denise D., und William T. Bielby. 1996. Women and men in film: Gender inequality among writers in a culture industry. Gender and Society 10:248-270.

Bikhchandani, Sushil, David Hirshleifer und Ivo Welch. 1992. A theory of fads, fashion, custom, and cultural change as informational cascades. Journal of Political Economy 100:992-1026.

Blossfeld, Hans-Peter. 2009. Educational assortative marriage in comparative perspective. Annual Review of Sociology 35:513-530.

Blossfeld, Hans-Peter, und Andreas Timm. 1997. Das Bildungssystem als Heiratsmarkt. Eine Längsschnittanalyse der Wahl von Heiratspartnern im Lebenslauf. Kölner Zeitschrift für Soziologie und Sozialpsychologie 53:440-476.

Boltanski, Luc, und Eve Chiapello. 2003. Der neue Geist des Kapitalismus. Konstanz: Universitäts Verlag.

Bothner, Matthew, Richard Haynes, Wonjae Lee und Edward N. Smith. 2010. When do Matthew effects occur? Journal of Mathematical Sociology 34:80-114.

Borghans, Lex, und Loek Groot. 1998. Superstardom and monopolistic power: Why media stars earn more than their marginal contribution to welfare. Journal of Institutional and Theoretical Economics 154:546-571.

Bourdieu, Pierre. 1985. The forms of capital. In Handbook of theory and research for the sociology of education, Hrsg. John G. Richardson, 241-258. New York: Greenwood.

Bröckling, Ulrich. 2007. Das unternehmerische Selbst. Soziologie einer Subjektivierungsform. Frankfurt a. M.: Suhrkamp.

Bührmann, Andrea D., Nina Wild, Marko Heyse und Thomas Dierschke. 2010. Viel Ehre, aber kaum Verdienst. Erhebung zur Arbeits- und Lebenssituation von Schauspielerinnen und Schauspielern in Deutschland. Methodenbericht. Universität Münster.

Bührmann, Andrea D., und Thomas Dierschke. 2012. Flexibel ohne Absicherung. Arbeitslosigkeit unter Filmschaffenden. IfS Working Papers Nr. 3. Münster.

Bührmann, Andrea D., Thomas Dierschke, Marko Heyse und Nina Wild. 2013. Die soziale Lage von Film- und Fernsehschauspieler/innen in Deutschland. IfS Working Papers Nr. 4. Münster.

Burt, Ronald S. 1992. Structural holes. The social structure of competition. Cambridge: Harvard University Press.

Carroll, Glenn R., und Anand Swaminathan. 2000. Why the microbrewery movement? Organizational dynamics of resource partitioning in the US brewing industry. American Journal of Sociology 106:715-762.

Carroll, Glenn R., Stanislav D. Dobrev und Anand Swaminathan. 2002. Organizational processes of resource partitioning. Research in Organizational Behavior 24:1-40. 
Cattani, Gino, und Simone Ferriani. 2008. A Core/periphery perspective on individual creative performance: Social networks and cinematic achievements in the Hollywood film industry. Organization Science 19:824-844.

Caves, Richard E. 2000. Creative industries: Contracts between art and commerce. Cambridge: Harvard University Press.

Cowen, Tyler. 2000. What price fame. Cambridge: Harvard University Press.

De Vaan, Mathijs, Balzazs Vedres, und David Stark. 2011. Disruptive diversity and recurring cohesion: Assembling creative teams in the video game industry, 1979-2009. Institute for Social and Economic Research and Policy at Columbia University.

Diekmann, Andreas, Matthias Näf und Manuel Schubiger. 2011. Die Rezeption (Thyssen-) preisgekrönter Artikel in der „Scientific Community“. Kölner Zeitschrift für Soziologie und Sozialpsychologie 64:563-581.

DiPrete, Thomas A., und Gregory M. Eirich. 2006. Cumulative advantage as a mechanism for inequality: A review of theoretical and empirical developments. Annual Review of Sociology 32:271-297.

DiPrete, Thomas A., Gregory M. Eirich und Matthew Pittinsky. 2010. Compensation benchmarking, leapfrogs, and the surge in executive pay. American Journal of Sociology 115:1671-1712.

Dobrev, Stanislav D., Tai-Young Kim und Michael T. Hannan. 2001. Dynamics of niche width and resource partitioning. The American Journal of Sociology 106:1299-1337.

Eikhof, Doris Ruth, und Axel Haunschild. 2006. Lifestyle meets market: Bohemian entrepreneurs in creative industries. Creativity and Innovation Management 15:234-241.

Eikhof, Doris Ruth, und Axel Haunschild. 2007. For art's sake! Artistic and economic logics in creative production. Journal of Organizational Behavior 28:523-538.

England, Paula. 2005. Gender inequality in labor markets: The role of motherhood and segregation. Social Politics: International Studies in Gender, State \& Society 12:264-288.

Faulkner, Robert R., und Andy B. Anderson. 1987. Short-term projects and emergent careers: Evidence from Hollywood. American Journal of Sociology 92:879-909.

Ferriani, Simone, Gino Cattani und Charles Baden-Fuller. 2009. The relational antecedents of project-entrepreneurship: Network centrality, team composition and project performance. Research Policy 38:1545-1558.

Florida, Richard. 2002. The rise of the creative class: And how it's transforming work, leisure, community and everyday life. New York: Basic Books.

Franck, Egon, und Stephan Nüesch. 2012. Talent and/or popularity: What does it take to be a superstar? Economic Inquiry 50:202-216.

Frank, Robert H., und Philip J. Cook. 1995. The Winner-take-all Society: How more and more Americans compete for ever fewer and bigger prizes, encouraging economic waste, income inequality, and an impoverished cultural life. New York: Free Press.

Girard, Monique, und David Stark. 2002. Distributing intelligence and organizing diversity in newmedia projects. Environment and Planning A 34:1927-1949.

Giuffre, Katherine. 1999. Sandpiles of opportunity: Success in the art world. Social Forces $77: 815-832$.

Gladwell, Malcolm. 2008. Outliers. The story of success. New York: Little, Brown and Company.

Godechot, Olivier. 2008. „Hold-up“ in finance: The conditions of possibility for high bonuses in the financial industry. Revue Francaise De Sociologie 49:95-123.

Godechot, Olivier. 2012. Is finance responsible for the rise in wage inequality in France? SocioEconomic Review 10:447-470.

Gottschall, Karin. 1999. Freie Mitarbeit im Journalismus. Zur Entwicklung von Erwerbsformen zwischen selbständiger und abhängiger Beschäftigung. Kölner Zeitschrift für Soziologie und Sozialpsychologie 51:635-654.

Gould, Roger V. 2002. The origins of status hierarchies: A formal theory and empirical test. The American Journal of Sociology 107:1143-1178. 
Granovetter, Mark S. 1973. The strength of weak ties. American Journal of Sociology 78:1360-1380.

Granovetter, Mark S. 1974. Getting a job. A study of contacts and careers. Cambridge: Harvard University Press.

Granovetter, Mark S. 1978. Threshold models of collective behavior. American Journal of Socio$\log y$ 83:1420-1443.

Grugulis, Irena, und Dimitrinka Stoyanova. 2012. Social capital and networks in film and tv: Jobs for the boys? Organization Studies 33:1311-1331.

Hacker, Jacob S., und Paul Pierson. 2010. Winner-Take-All politics: Public policy, political organization, and the precipitous rise of top incomes in the United States. Politics \& Society 38:152-204.

Hannan, Michael T., und John Freeman. 1977. Population ecology of organizations. American Journal of Sociology 82:929-964.

Hellmann, Kai-Uwe. 2003. Soziologie der Marke. Frankfurt a. M.: Suhrkamp.

Henninger, Annette, und Karin Gottschall. 2007. Freelancers in Germany's old and new media industry: Beyond standard patterns of work and life? Critical Sociology 33:43-71.

Hsu, Greta. 2006. Jacks of all trades and masters of none: Audiences' reactions to spanning genres in feature film production. Administrative Science Quarterly 51:420-450.

Hsu, Greta, Michael T. Hannan und Özgecan Koçak. 2009. Multiple category memberships in markets: An integrated theory and two empirical tests. American Sociological Review 74:150-169.

Hutter, Michael. 2011. Infinite surprises. On the stabilization of value in the creative industries. In The worth of goods. Valuation and pricing in the economy, Hrsg. Jens Beckert und Patrik Aspers, 201-220. Oxford: Oxford University Press.

Jones, Candace. 1996. Careers in project networks: The case of the film industry. In The boundaryless career: A new employment principle for a new organizational era, Hrsg. Michael B. Arthur und Denise M. Rousseau, 58-75. Oxford: Oxford University Press.

Karpik, Lucien. 2010. Valuing the unique: The Economics of Singularities. Princeton: Princeton University Press.

Keuschnigg, Marc. 2012a. Konformität durch Herdenverhalten. Theorie und Empirie zur Entstehung von Bestsellern. Kölner Zeitschrift für Soziologie und Sozialpsychologie 64:1-36.

Keuschnigg, Marc. 2012b. Das Bestseller-Phänomen. Die Entstehung von Nachfragekonzentration im Buchmarkt. Wiesbaden: Springer VS.

Koppetsch, Cornelia. 2006. Zwischen Disziplin und Expressivität. Zum Wandel beruflicher Identitäten im neuen Kapitalismus. Berliner Journal für Soziologie 16:155-172.

Koppetsch, Cornelia. 2008. Der Markt der Ideen. Neue Wege der Professionalisierung am Beispiel der Kultur- und Medienindustrien. Soziale Welt 4:327-350.

Lazear, Edward P., und Sherwin Rosen. 1981. Rank-order tournaments as optimum labor contracts. Journal of Political Economy 89:841-864.

Leaver, Adam. 2010. A different take: Hollywood's unresolved business model. Review of International Political Economy 17:454-480.

Levy, Emanuel. 1989. The democratic elite: America's movie stars. Qualitative Sociology 12:29-54.

Lincoln, Anne E., und Michael Patrick Allen. 2004. Double jeopardy in Hollywood: Age and gender in the careers of film actors, 1926-1999. Sociological Forum 19:611-631.

Lutter, Mark. 2010. Zur Erklärung von Diffusionsprozessen. Das Beispiel der Einführung staatlicher Lotterien in den USA. Zeitschrift für Soziologie 39:363-381.

Lutter, Mark. 2012a. Anstieg oder Ausgleich? Die multiplikative Wirkung sozialer Ungleichheiten auf dem Arbeitsmarkt für Filmschauspieler. Zeitschrift für Soziologie 41:435-457.

Lutter, Mark. 2012b. Tagträume und Konsum: Die imaginative Qualität von Gütern am Beispiel der Nachfrage für Lotterien. Soziale Welt 63:233-251.

Lutter, Mark. 2013. Is there a closure penalty? Cohesive network structures, diversity, and gender inequalities in career advancement. MPIfG Discussion Paper 13/9. Köln: Max-Planck-Institut für Gesellschaftsforschung. 
Lynn, Freda B., Joel M. Podolny und Lin Tao. 2009. A sociological (de) construction of the relationship between status and quality. American Journal of Sociology 115:755-804.

MacDonald, Glenn M. 1988. The economics of rising stars. The American Economic Review 78:155-166.

March, James G., und Robert I. Sutton. 1997. Crossroads - organizational performance as a dependent variable. Organization Science 8:698-706.

Mathieu, Chris. Hrsg. 2012. Careers in creative industries. New York: Routledge.

McCall, Leslie, und Christine Percheski. 2010. Income inequality: New trends and research directions. Annual Review of Sociology 36:329-347.

McKinlay, Alan, und Chris Smith. Hrsg. 2009. Creative labour. Working in the creative industries. Basingstoke: Palgrave Macmillan.

McPherson, Miller, Lynn Smith-Lovin und James M. Cook. 2001. Birds of a feather: Homophily in social networks. Annual Review of Sociology 27:415-444.

Meier, Henk Erik. 2008. Institutional complementarities and institutional dynamics: Exploring varieties in European football capitalism. Socio-Economic Review 6:99-133.

Menger, Pierre-Michel. 1999. Artistic labor markets and careers. Annual Review of Sociology 25:541-574.

Menger, Pierre-Michel. 2009. Le travail créateur. S'accomplir dans l'incertain. Paris GallimardSeuil-Éditions de l'EHESS.

Merton, Robert King. 1968. The Matthew effect in science. Science 159:56-63.

Müller-Jentsch, Walther. 2005. Künstler und Künstlergruppen. Soziologische Ansichten einer prekären Profession. Berliner Journal für Soziologie 15:159-177.

Münch, Richard. 2010. Der Monopolmechanismus in der Wissenschaft. Auf den Schultern von Robert K. Merton. Berliner Journal für Soziologie 20:341-370.

Neckel, Sighard. 2001. „Leistung“ und „Erfolg“. Die symbolische Ordnung der Marktgesellschaft. In Gesellschaftsbilder im Umbruch, Hrsg. Hans Peter Müller Eva Barlösius und Steffen Sigmund, 245-268. Opladen: Leske und Budrich.

Neckel, Sighard. 2008. Flucht nach vorn. Die Erfolgskultur der Marktgesellschaft. Frankfurt a. M.: Campus.

Neckel, Sighard. 2010. Refeudalisierung der Ökonomie: Zum Strukturwandel kapitalistischer Wirtschaft. MPIfG Working Paper 10/6.

Neckel, Sighard, und Kai Dröge. 2003. Die Verdienste und ihr Preis. Leistung in der Marktgesellschaft. In Befreiung aus der Mündigkeit. Paradoxien des gegenwärtigen Kapitalismus, Hrsg. Axel Honneth, 93-116. Frankfurt a. M.: Campus.

Neckel, Sighard, Ana Mijic, Christian von Scheve und Monica Tritton. Hrsg. 2010. Sternstunden der Soziologie. Wegweisende Theoriemodelle des soziologischen Denkens. Frankfurt a. M.: Campus.

Nicolai, Alexander, und Alfred Kieser. 2002. Trotz eklatanter Erfolglosigkeit: Die Erfolgsfaktorenforschung weiter auf Erfolgskurs. Die Betriebswirtschaft 62:579-596.

Pager, Devah, und Hana Shepherd. 2008. The sociology of discrimination: Racial discrimination in employment, housing, credit, and consumer markets. Annual Review of Sociology 34:181-209.

Park, D. Y., und J. M. Podolny. 2000. The competitive dynamics of status and niche width: US investment banking, 1920-1949. Industrial and Corporate Change 9:377-414.

Perretti, Fabrizio, und Giacomo Negro. 2006. Filling empty seats: How status and organizational hierarchies affect exploration versus exploitation in team design. The Academy of Management Journal 49:759-777.

Perretti, Fabrizio, und Giacomo Negro. 2007. Mixing genres and matching people: A study in innovation and team composition in Hollywood. Journal of Organizational Behavior 28:563-586.

Petersen, Trond, und Ishak Saporta. 2004. The opportunity structure for discrimination. American Journal of Sociology 109:852-901. 
Phillips, Damon J., und Ezra W. Zuckerman. 2001. Middle-status conformity: Theoretical restatement and empirical demonstration in two markets. American Journal of Sociology 107:379-429.

Podolny, Joel M. 2001. Networks as the pipes and prisms of the market. American Journal of Sociology 107:33-60.

Podolny, Joel. 2005. Status signals. A sociological study of market competition. Princeton: Princeton University Press.

Rao, Hayagreeva, Philippe Monin und Rodolphe Durand. 2003. Institutional change in toque ville: Nouvelle cuisine as an identity movement in French gastronomy. American Journal of Sociology 108:795-843.

Rosen, Sherwin. 1981. The economics of superstars. American Economic Review 71:845-858.

Rosen, Sherwin. 1986. Prizes and incentives in elimination tournaments. American Economic Review 76:701-715.

Rössel, Jörg. 2009. Kulturelles Kapital und Musikrezeption. Eine empirische Überprüfung von Bourdieus Theorie der Kunstwahrnehmung. Soziale Welt 60:239-255.

Rössel, Jörg, und Kathi Bromberger. 2009. Strukturiert kulturelles Kapital auch den Konsum von Populärkultur? Zeitschrift für Soziologie 38:494-513.

Rossman, Gabriel. 2012. Climbing the charts: What radio airplay tells us about the diffusion of innovation. Princeton: Princeton University Press.

Rossman, Gabriel, Nicole Esparza und Phillip Bonacich. 2010. I'd like to thank the academy, team spillovers, and network centrality. American Sociological Review 75:31-51.

Rost, Katja. 2010. The rise in executive compensation - consequence of a ,War for Talents"? Working paper. University of Zurich. Institute for Organization and Administrative Science.

Rost, Katja. 2011. The strength of strong ties in the creation of innovation. Research Policy 40:588-604.

Seeliger, Martin. 2012. Deutscher Gangstarap. Zwischen Affirmation und Empowerment. Berlin: Posth.

Skopek, Jan, Florian Schulz und Hans-Peter Blossfeld. 2011. Who contacts whom? Educational homophily in online mate selection. European Sociological Review 27:180-195.

Stark, David. 2009. The sense of dissonance: Accounts of worth in economic life. Princeton: Princeton University Press.

Szydlik, Marc. 1999. Erben in der Bundesrepublik Deutschland: Zum Verhältnis von familialer Solidarität und sozialer Ungleichheit. Kölner Zeitschrift für Soziologie und Sozialpsychologie 51:80-104.

Szydlik, Marc. 2004. Inheritance and inequality: Theoretical reasoning and empirical evidence. European Sociological Review 20:31-45.

Szydlik, Marc, und Jürgen Schupp. 2004. Wer erbt mehr? Erbschaften, Sozialstruktur und Alterssicherung. Kölner Zeitschrift für Soziologie und Sozialpsychologie 56:609-629.

Tilly, Charles. 1998. Durable inequality. Berkeley: University of California Press.

Uzzi, Brian, und Jarrett Spiro. 2005. Collaboration and creativity: The small world problem. American Journal of Sociology 111:447-504.

Van de Rijt, Arnout, Eran Shor, Charles Ward und Steven Skiena. 2013. Only 15 minutes? The social stratification of fame in printed media. American Sociological Review 78:266-289.

Vedres, Balázs, und David Stark. 2010. Structural folds: Generative disruption in overlapping groups. American Journal of Sociology 115:1150-1190.

Western, Bruce, Deirdre Bloome und Christine Percheski. 2008. Inequality among American families with children, 1975 to 2005. American Sociological Review 73:903-920.

Zuckerman, Ezra W., Tai-Young Kim, Kalinda Ukanwa und James von Rittmann. 2003. Robust identities or nonentities? Typecasting in the feature-film labor market. American Journal of Sociology 108:1018-1074.

Zuckerman, Harriet. 2010. Dynamik und Verbreitung des Matthäus-Effekts. Berliner Journal für Soziologie 20:309-340. 
Mark Lutter, 1976, Dr. rer. pol., wissenschaftlicher Mitarbeiter am Max-Planck-Institut für Gesellschaftsforschung in Köln. Forschungsgebiete: Wirtschafts- und Organisationssoziologie; Politische Soziologie; Soziale Ungleichheit und Sozialstrukturanalyse; Methoden empirischer Sozialforschung. Veröffentlichungen: Supranational Cultural Norms, Domestic Value Orientations and the Diffusion of Same-sex Union Rights in Europe, 1988-2009, International Sociology 28, 2013 (mit Juan J. Fernández); Anstieg oder Ausgleich? Die multiplikative Wirkung sozialer Ungleichheiten auf dem Arbeitsmarkt für Filmschauspieler. Zeitschrift für Soziologie 41, 2012; Why the Poor Play the Lottery. Sociological Approaches to Explaining Class-based Lottery Play, Sociology, online first, December 17, 2012 (mit J. Beckert). 